\title{
Book Review-Livre nouveau
}

G.A. Glazer: Hémodynamique de la circulation systématique dans l'hyper-tension artérielle (en russe). Meditsina, Moscou 1970. 1 vol., 176 pp., 15 fig., 41 tab.; 89 Kopeks, broché.

Dans cet excellent travail d'investigation clinique chez Thomme, G.A. Glazer a étudié la volémie, le debit cardiaque (mesuré par les courbes de dilution du bleu Evans) et le debit sanguin renal dans différents types d'hypertension artérielle.

Parmi ses constatations les plus originales, on retiendra (a) Гaugmentation nette de la volémie et de $\Gamma$ index cardiaque dans la sténose de l'isthme aortique, (b) le fait que, dans le syndrome de Takayashu, l'augmentation de la volémie et de $\Gamma$ index cardiaque qui existent en Tabsence de sténose artérielle rénale font place à une diminution de $\Gamma$ index cardiaque avec augmentation des resistances périphériques lorsqu'il y a une sténose artérielle rénale, (c) une correlation statistiquement significative entr secretion d'aldostérone et resistances périphériques, et (d) le carac-tère tardif de la diminution du debit sanguin renal.

L'influence du traitement hypotenseur fait apparaître, sous Guanéthidine, une augmentation de la volémie que Tauteur rapporte à une insuffisance cardiaque.

On ne peut que regretter que cet excellent travail, écrit entièrement en russe, ne comporte même pas de resume anglais, car il aurait mérité une audience Inter nationale. P.Y. Hatt 